\title{
COVID Blindness: Delayed Diagnosis of Aseptic Meningitis in the COVID-19 Era
}

\author{
Taku Harada $^{1,2}$, Takashi Watari ${ }^{3}$, Taiju Miyagami $^{4}$, Satoshi Watanuki ${ }^{5}$, Taro Shimzu² $^{2}$ Juichi Hiroshige $^{1}$ \\ ${ }^{1}$ Division of General Medicine, Showa University Koto Toyosu Hospital, Toyosu Koto-ku, Japan \\ ${ }^{2}$ Division of Diagnostic and Generalist Medicine, Dokkyo Medical University Hospital, Mibu-cho, Japan \\ ${ }^{3}$ Postgraduate Clinical Training Centre, Shimane University Hospital, Enya-cho, Japan \\ ${ }^{4}$ Department of General Medicine, Juntendo University Faculty of Medicine, Bunkyo-ku, Japan \\ ${ }^{5}$ Division of Emergency and General Medicine, Tokyo Metropolitan Tama Medical Center, Fuchu-shi, Japan
}

Doi: 10.12890/2020_001940 - European Journal of Case Reports in Internal Medicine - () EFIM 2020

Received: $10 / 08 / 2020$

Accepted: $14 / 08 / 2020$

Published: $23 / 10 / 2020$

How to cite this article: Harada T, Watari T, Miyagami T, Watanuki S, Shimzu T, Hiroshige J. COVID Blindness: delayed diagnosis of aseptic meningitis in the COVID-19 era. EJCRIM 2020;7: doi:10.12890/2020_001940.

Conflicts of Interests: The Authors declare that there are no competing interests.

This article is licensed under a Commons Attribution Non-Commercial 4.0 License

\section{ABSTRACT}

Diagnostic errors are a serious problem in healthcare. The diagnostic process is highly susceptible to cognitive bias and the current COVID-19 pandemic may cause normally accurate healthcare workers to make incorrect decisions. We report a case of aseptic meningitis that required five healthcare visits before it was correctly diagnosed. This case highlights the risk of anchoring bias and the importance of carefully assessing diagnostic processes during the COVID-19 pandemic.

\section{LEARNING POINTS}

- COVID-19 can disrupt the healthcare system and clinical environment and affect diagnosis due to anchoring bias.

- Healthcare providers should carefully check the COVID-19 illness script to reduce diagnostic errors.

- Healthcare providers should prepare and practice a diagnostic debiasing strategy during the COVID-19 pandemic.

\section{KEYWORDS}

COVID-19, COVID blindness, diagnostic error

\section{INTRODUCTION}

Diagnostic errors are a serious problem in healthcare. The need for timely and accurate diagnosis is especially important in light of the surge in coronavirus (COVID-19) cases during the pandemic ${ }^{[1]}$. However, studies have reported diagnostic errors and biases associated with COVID-19. Gandhi et al. classified the risk of COVID-19-related diagnostic errors into eight categories, including errors in diagnosing COVID-19, errors due to the impact of the pandemic on the healthcare system and clinical environment, and delays in the diagnosis and treatment of other diseases ${ }^{[1]}$. This pandemic is leading to anchoring bias in healthcare providers that affects diagnostics, resulting in the delayed diagnosis of other diseases, especially in patients presenting with acute fever or respiratory distress ${ }^{[2]}$. Here, we discuss the case of a patient with aseptic meningitis, who required five healthcare visits before he was correctly diagnosed. He was repeatedly suspected of having COVID-19 despite the lack of lower respiratory tract symptoms or decreased saturated oxygen in arterial blood (SpO ${ }_{2}$ ) levels. We also discuss the impact of the COVID-19 epidemic on the diagnostic process. 


\section{CASE DESCRIPTION}

A 20-year-old man presented to the emergency department with a 9-day history of fever and worsening headache. He had previously visited his primary care physician after developing fever and was prescribed paracetamol. Four days before the hospital visit, the patient developed a headache, which persisted with no improvement in his condition. One day before the visit, he again consulted his primary care physician and was referred to the hospital because of suspected COVID-19.

On his first visit, the patient complained of a frontal pulsatile headache, but there was no nasal discharge, sore throat, cough, sputum, dyspnoea, olfactory disturbance, taste disturbance, abdominal symptoms or diarrhoea. He had no medical or travel history, and no close contacts had developed fever or illness. His physical examination was normal, and chest radiography and blood tests revealed no major abnormalities. The PCR test was negative for severe acute respiratory syndrome coronavirus-2 (SARS-CoV-2), and he was discharged and advised to follow-up with healthcare workers. However, his fever and headache worsened, and his physician referred him to the emergency department.

On examination, his body temperature was $37.5^{\circ} \mathrm{C}$, respiration rate was 16 breaths $/ \mathrm{min}, \mathrm{SpO}_{2}$ was $98 \%$ (room air), and physical examination was positive for nuchal rigidity and jolt accentuation. Blood tests revealed a glucose level of $107 \mathrm{mg} / \mathrm{dl}$, C-reactive protein level of $0.68 \mathrm{mg} /$ $\mathrm{dl}$, and normal blood cell count, electrolyte levels, and liver and kidney function. A lumbar puncture was performed, and the cerebrospinal fluid analysis showed a pressure of $35 \mathrm{cmH}_{2} \mathrm{O}$, cell count of 98 cells/ $/ \mathrm{l}$ (mononuclear cells $88 \%$, polymorphic cells $12 \%$ ), total protein of 88 $\mathrm{mg} / \mathrm{dl}$, and glucose level of $65 \mathrm{mg} / \mathrm{dl}$. The patient was finally diagnosed with aseptic meningitis. The spinal fluid culture and PCR results for varicella-zoster and herpes simplex viruses were negative. The patient was discharged after 10 days with a good clinical course.

\section{DISCUSSION}

Diagnostic mistakes are a common cause of medical errors in healthcare, resulting in serious complications, including death. With the number of patients rapidly increasing due to the COVID-19 pandemic, there is an urgent need for timely and accurate diagnosis ${ }^{[1]}$. However, few studies have described the diagnostic errors and biases associated with COVID-19. These errors include mistakes in diagnosing COVID-19 and errors arising from overemphasis on COVID-19. COVID-19 has had an impact on the timely diagnosis and treatment of other diseases. For example, diagnostic errors have been made in relation to other acute illnesses and there have been delays in seeking medical consultation due to fear of COVID-19, irrespective of the type of the acute or chronic illness ${ }^{[1]}$. 'COVID blindness' is similar to anchoring bias. It refers to the bias of the healthcare provider to diagnose COVID-19 in patients with typical symptoms, such as acute fever or respiratory distress, without considering alternative diagnoses ${ }^{[2]}$. This will affect us severely during the ongoing pandemic. Ways to reduce COVID-19related diagnostic errors include using debiasing strategies, introducing 'diagnostic huddles' or time-out to reflect on the diagnostic process, improving the clinical/hospital environment, and using technology ${ }^{[1]}$. Furthermore, recent reports have suggested that reinforcement of the illness script is effective in reducing diagnostic errors ${ }^{[3]}$. This intervention is directed at increasing knowledge of clinical findings that discriminate between similar-looking diseases. COVID-19 is an emerging infectious disease that can disrupt the healthcare system and threaten the psychological and physical safety of healthcare workers. Healthcare workers, especially during COVID-19, should be aware of any bias influencing their diagnostic process and assessment of the consistency of the clinical course with COVID-19.

The initial symptoms of COVID-19 may vary, but the clinical course leading to pneumonia often follows a typical timeline. It takes an average of 5 days until the patient develops respiratory distress and 7 days until hospitalization ${ }^{[4]}$.

One study suggested how COVID-19 might complicate meningitis, which is almost always associated with encephalitis, as meningitis rarely occurs alone ${ }^{[4]}$. The incidence of headache in COVID-19 is $6.5 \%{ }^{[5]}$. The main complaints of our patient were fever and headache, and it was reasonable to consider COVID-19 as a differential diagnosis, considering the ongoing pandemic. However, the clinical course of worsening headache without dyspnoea or decreased $\mathrm{SpO}_{2}$ with a fever lasting 8 days is atypical for COVID-19. Instead of completing the diagnostic process by confirming a negative chest $x$-ray or PCR, other alternative diagnoses should have been considered. This was a clear example of COVID blindness.

The COVID-19 epidemic threatens not only the physical and psychological safety of healthcare providers, but also the healthcare system itself, which may affect clinical diagnosis and decision-making ${ }^{[1]}$. Healthcare providers fighting the pandemic may develop an anchoring bias. The probability of COVID-19 is high in a patient with fever or respiratory distress during the epidemic. However, we must rely on our foundational training and carefully consider the differential diagnosis, especially in patients with atypical clinical courses. 


\section{REFERENCES}

1. Gandhi TK, Singh H. Reducing the risk of diagnostic error in the COVID-19 era. J Hosp Med 2020;15(6):363-366

2. Brown L. COVID blindness. Diagnosis (Berl) 2020;7(2):83-84.

3. Mamede S, de Carvalho-Filho MA, de Faria RMD, Franci D, Nunes MPT, Ribeiro LMC, et al. 'Immunizing' physicians against availability bias in diagnostic reasoning: a randomized controlled experiment. BMJ Qual Saf 2020;29(7):550-559.

4. Romoli M, Jelcic I, Bernard-Valnet R, Garcia Azorin D, Mancinelli L, Akhvlediani T, et al. A systematic review of neurological manifestations of SARS-CoV-2 infection: the devil is hidden in the details. Eur J Neurol 2020 Jun 5;10.1111/ene.14382. doi: 10.1111/ene.14382. Online ahead of print.

5. Wang D, Hu B, Hu C, Zhu F, Liu X, Zhang J, et al. Clinical characteristics of 138 hospitalized patients with 2019 novel coronavirus-infected pneumonia in Wuhan, China. JAMA 2020;323(11):1061-1069. 